PART II

RESOLUTIONS ADOPTEES 


\section{RESOLUTIONS ADOPTEES}

\section{Comite éxécutif}

(a) Que l'article V, ro des Statuts soit modifié comme suit:

Les présents Statuts entreront en vigueur au moment de leur adoption par l'Assemblée générale et seront valables jusqu'au 3 I décembre I958. Après cette date, ils seront renouvelés pour une autre période de douze ans, sauf avis contraire des organismes adhérents.

(b) Que l'article I, I du Règlement soit modifié comme suit:

L'Union se compose de membres désignés par le Comité exécutif en raison de leur activité dans une branche de l'Astronomie, et sur proposition des organisations adhérentes. Les Commissions permanentes peuvent proposer la cooptation de personnalités appartenant à des pays qui n'adhèrent pas à l'Union. Les changements à apporter à la liste des membres sont soumis par le Comité exécutif à la Commission des Présentations, composée d'un représentant de chaque pays adhérent, désigné par l'organisme adhérent.

Le Secrétaire général publie la liste des membres après chaque Assemblée générale.

(c) Que l'article II, 5 du Règlement soit modifié comme suit:

Toute proposition à soumettre à l'Assemblée générale doit être reçue par le Secrétaire général au moins cinq mois avant l'ouverture de l'Assemblée générale. Toute proposition soumise par un organisme adhérent, par un Comité national d'astronomie, par une commission de l'Union, ou par une Commission mixte, affiliée à l'Union, et reçue par le Secrétaire général cinq mois en avance, doit être inscrite à l'ordre du jour.

(d) Que l'article III, Io du Règlement soit modifié comme suit:

Le Comité exécutif de l'Union comprend un Président, six Vice-Présidents au plus et un Secrétaire général, élus par l'Assemblée générale.

$(\varepsilon)$ Que l'article IV, I5 du Règlement soit modifié comme suit:

Le Président et les membres de chaque commission sont élus par l'Assemblée générale sur proposition du Comité exécutif de l'Union; ils sont choisis parmi les membres de l'Union. Ils exercent leur mandat jusqu'à la fin de l'Assemblée générale ordinaire suivante et sont rééligibles.

Lorsqu'une commission comprend des membres désignés en partie par l'Union Astronomique et en partie par une autre Union rattachée au Conseil International des Unions Scientifiques, elle a la faculté d'élire elle-même son Président.

Les commissions établissent elles-mêmes leur règlement d'ordre intérieur, elles peuvent s'adjoindre de nouveaux membres par cooptation et à la majorité des deux tiers de voix. Si les nouveaux membres n'appartiennent pas à des pays adhérents, ils ne prennent part aux travaux de la commission et de l'Assemblée générale qu'à titre consultatif.

$(f)$ Que l'article VII, 22 et 23 du Règlement soit modifié comme suit:

22. Les Transactions de l'Union sont fournies gratuitement:

(i) aux membres de l'Union;

(ii) aux observatoires dont la liste est établie par le Comité exécutif;

(iii) aux organisations adhérentes et comités nationaux.

23. Le Comité exécutif décide des modalités de distribution des autres publications.

24. Les membres de l'Union peuvent acquérir les publications à prix réduit.

(g) Le Comité exécutif propose de réserver une somme de rooo dollars par an pour les voyages des jeunes astronomes lors de la prochaine Assemblée générale. 
La Commission de Finance propose à l'Assemblée générale:

I. Que les subventions suivantes soient allouées pour la période s'écoulant du Ier janvier I956 au 3I décembre I958:

\section{(a) Subventions ANNUElles}

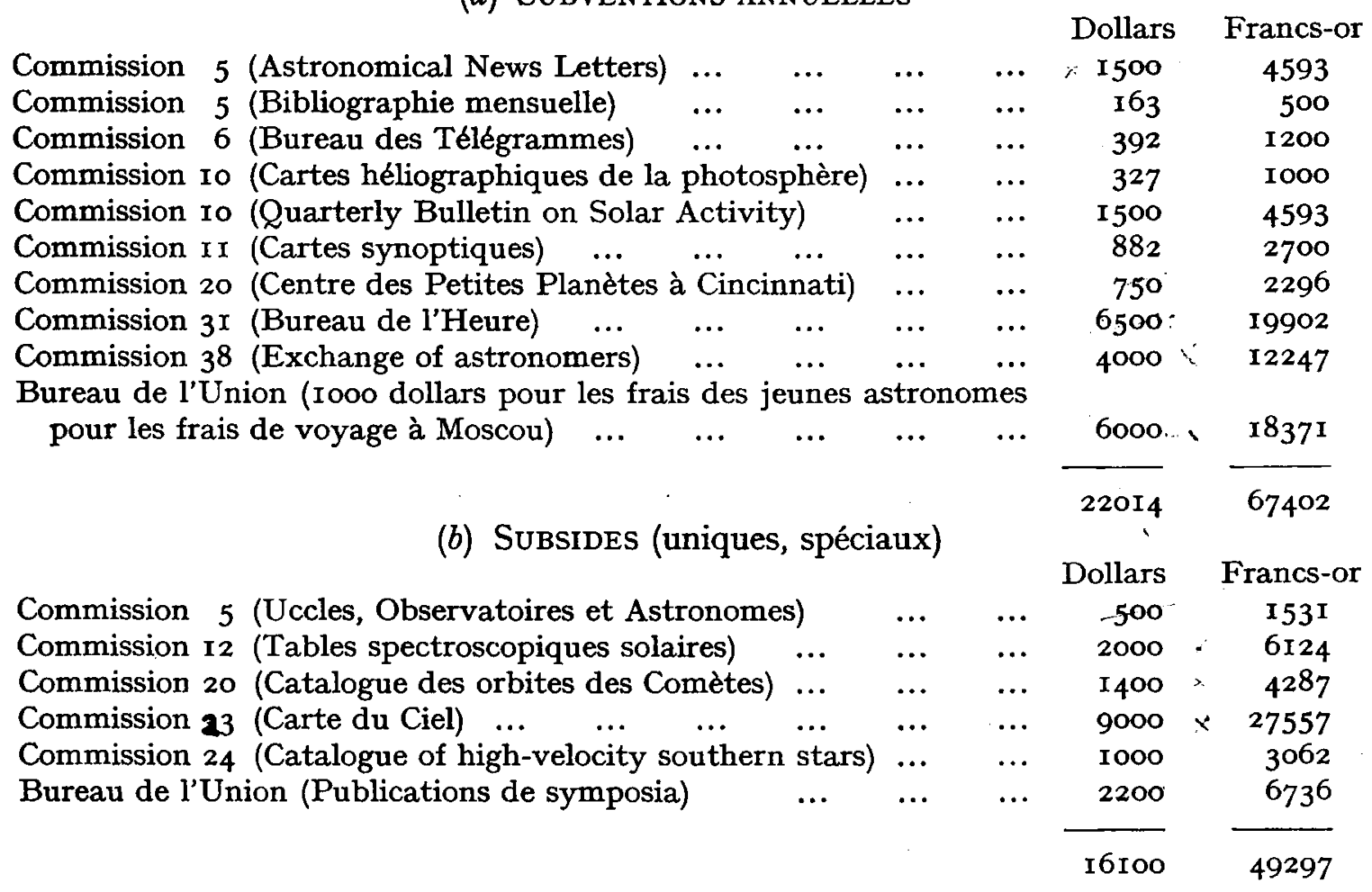

2. Que l'unité de cotisation pour les années 1956 , I957, I958 reste fixée à 500 francs-or.

\section{Commission 3}

\section{RECOMMANDATIONS}

I. La Commission recommande la notation $\mathrm{cm} \cdot .^{-1}$ pour le Kayser.

2. La Commission recommande l'emploi de la notation A pour l'angstrom toutes les fois que la confusion avec une autre grandeur est impossible et l'emploi de la notation $A$ dans les autres cas.

3. La Commission a recommandé que pour l'abréviation des noms des constellations soit définitivement adoptée la forme à trois lettres.

4. La Commission recommande l'emploi des symboles figurant dans le tableau de la p. 23 du Draft Report. On tiendra compte que, par suite d'une erreur typographique à la première ligne, la lettre $\kappa$ (kappa) doit être remplacée par $r$. Il sera précisé qu'à la dernière ligne la composante $\Pi$ (grand pi) de la vitesse doit toujours être comptée positivement vers l'extérieur. (Voir aussi page 78 de ce volume.)

\section{Commission 5}

I. La Commission 5 prie instamment les institutions et les personnes publiant un travail concernant l'astronomie de bien vouloir en faire parvenir au moins un exemplaire, aussi rapidement que possible et sans passer par les lents services généraux de distribution, aux adresses ci-après, des bibliographies astronomiques.

(I) The Editor of the Astronomischer Jahresbericht. Astronomisches Rechen-Institut, I4 Grabengasse, Heidelberg, Germany. 